\title{
Expression of HOXA-10 and HOXA-11 in the endometria of women with idiopathic infertility
}

\author{
Malgorzata Szczepańska1, Przemyslaw Wirstlein ${ }^{1}$, Michał Luczak², Paweł P. Jagodzinski², \\ Jana Skrzypczak ${ }^{1}$
}

${ }^{1}$ Department of Obstetrics, Gynecology and Gynecological Oncology, Division of Reproduction, K. Marcinkowski Medical University, Poznan, Poland

${ }^{2}$ Department of Biochemistry and Molecular Biology, Poznan University of Medical Sciences,

Poznan, Poland

\begin{abstract}
In fertile women, HOXA-10 and HOXA-11 expression rises during the luteal phase, with the peak occurring during the implantation window, and stays at a high level until the end of the cycle. We evaluated the transcript and protein levels of HOXA-10 and HOXA-11 in the endometria of patients with idiopathic infertility $(\mathrm{n}=15)$ and control patients $(\mathrm{n}=10)$. The amounts of mRNA were determined by reverse transcription and real-time quantitative PCR. The protein levels were evaluated by Western blotting analysis. Using immunohistochemical techniques, we compared the localization of HOXA-10 and HOXA-11 proteins in the implantation window between the study and control groups. We observed statistically significantly decreased HOXA-10 and HOXA-11 transcript levels ( $p=0.003, p=0.012$ respectively) in infertile patients compared to controls. There was no significant decrease in HOXA-10 protein levels between these groups $(p=0.074)$. However, we observed a significantly higher level of HOXA-11 protein in the endometria of infertile patients compared to controls $(\mathrm{p}=0.015)$. HOXA-10 and HOXA-11 proteins were localized in the nuclei of the endometrial stromal cells. Immunohistochemical analyses did not reveal differences between amounts of HOXA-10 and HOXA-11 protein levels in infertility and control groups. Our results suggest that HOXA-10 and HOXA-11 gene expression in the endometrium during the implantation window may not be altered in patients with idiopathic infertility. (Folia Histochemica et Cytobiologica 2011; Vol. 49, No. 1, pp. 111-118)
\end{abstract}

Key words: HOXA-10, HOXA-11, endometrium, idiopathic infertility

\section{Introduction}

Despite advanced and sophisticated diagnostic methods, $15 \%$ of the causes of infertility remain unknown. Research indicates that the number of pregnancies in this group is limited by the process of implantation [1]. An implantation of the blastocyst may occur only over a very short interval, known as the implantation window, i.e. 7-9 days after ovulation [2]. During that

Correspondence address: P. Wirstlein, Department of Obstetrics, Gynecology and Gynecological Oncology, Division of Reproduction, K. Marcinkowski Medical University, Polna Str. 33, 60-535 Poznan, Poland;

tel.: (+ 48 61) 84193 02;

e-mail: abys@wp.pl defined period, the endometrium reaches its maximum receptivity to embryo implantation and, consequently, pregnancy. Optimal implantation conditions are preceded by proliferation and differentiation of elements including endometrial glands, stroma, blood vessels, smooth muscle cells and fibroblasts. Impaired endometrial growth and differentiation may be a significant factor contributing to infertility [1]. Unfortunately, it is still the case that relatively little is known about the molecular mechanism which regulates endometrial development and, consequently, leads to optimal implantation conditions [2]. Earlier findings of our research group regarding endometrial receptivity in patients with infertility of different etiology and pregnancy loss suggest that altered expression of several molecules has a direct influence on implantation [3-7]. 
Transcription factors bind selectively to specific promoter DNA sequences and co-ordinate the process of cell differentiation by regulating their expression.

The most essential part of the transformation process leading to pregnancy is optimal endometrial receptivity. It is probable that transcription factors, whose expression in the endometrium is regulated by progesterone and stimulated by the embryo, may be crucial for implantation [8].

Protein products of the HOXA-10 and HOXA-11 homebox gene subclass are one of the best-known transcription factors participating in implantation [9].

The most vital implantation condition in mice is maternal expression of HOXA-10 and HOXA-11 [10, 11]. Transgenic mice without the genes in question are infertile due to implantation defects. Mice without the HOXA-10 and HOXA-11 expression have a normal number of embryos which may be implanted into the uterus of healthy subjects. The significance of HOXA-10 and its role in the implantation process has been confirmed by using HOXA- 10 antisense oligonucleotides. Transfer of HOXA-10 antisense oligonucleotides into the uteri of mice results in a significant decrease of embryo implantations.

The expression of HOXA-10 and HOXA- 11 genes could be also epigenetically regulated. The presence of a $\mathrm{CpG}$ island in the promotor sequence of both genes has previously been reported [12].

No HOXA gene mutations were found in women with congenital absence of the uterus and vagina [13]. HOXA-10 and HOXA-11 expression is present at the same level during the entire menstrual cycle and is regulated by estrogen and progesterone concentrations $[14,15]$. In normal fertile women, HOXA-10 and HOXA-11 expression rises significantly during the luteal phase, with the peak occurring during the implantation window, and stays at a high level until the end of the cycle [9]. Findings regarding the importance of HOXA-10 and HOXA-11 genes are contradictory. Some authors have reported a dramatic rise in the HOXA-10 and 11 expression level in the midluteal phase $[6,14,16]$, whereas other authors do not confirm this phenomenon [17].

The aim of our study was to determine whether endometrial expression of HOXA-10 and HOXA-11 genes, which are the source of transcription factors crucial for implantation, is normal in women with idiopathic infertility.

HOXA-10 and HOXA-11 genes expression, and the level of their protein products in the endometrium at the time of implantation window, were evaluated in a group of women with idiopathic infertility, and in a control group.

\section{Material and methods}

The study included 25 women of reproductive age, 15 of whom had idiopathic infertility. These 15 comprised the study group. The control group comprised ten normal fertile women. Women from both groups underwent laparoscopy, and the infertile women underwent hysteroscopy. Pipelle or hysteroscopic biopsy was used to acquire the endometrium from each woman during the implantation window, i.e. 7-9 days after ultrasound-confirmed ovulation. Subsequent histopathological study excluded women with a pathological endometrium from both groups.

The infertile group consisted of women with normal hysterosalpingography and hormonal test results and whose partners' infertility had been excluded. The control group included women who had at least one child, regular menses and no anatomical or functional changes in the endometrium. None of the women had been using oral contraception, hormonal therapy or an intrauterine device for at least six months prior to laparoscopy being performed. Patient characteristics are shown in Table 1.

\section{RNA isolation}

Part of the endometrium collected during the implantation window was transferred to histology. The remainder was divided in the following way: at least a $30 \mathrm{mg}$ piece was placed in RNAlater solution (Qiagen, Germany) and frozen at $-80^{\circ} \mathrm{C}$ until RNA isolation; whereas the rest of the endometrium was frozen in liquid nitrogen for Western blot protein analysis. The RNA isolation was conducted with the use of RNeasy Protect Mini Kit (Qiagen, Germany). We used an additional RNase-Free DNase Set recommended by the manufacturer (Qiagen, Germany) to eliminate the risk of DNA contamination.

\section{Reverse transcription}

For reverse transcription, we took $1 \mu \mathrm{g}$ of previously isolated RNA. Quantity at OD $260 \mathrm{~nm}$ and purity at OD 260/280 nm samples were checked spectrophotometrically with NanoDrop ND1000 (ThermoScientific, USA). Using QuantiTect Reverse Transcription Kit and oligodT (Qiagen, Germany) we obtained cDNA from previously isolated mRNA fraction. The reverse transcription reaction was antedated by additional elimination of DNA using QuantiTect Wipeout buffer, one of the components of QuantiTect Reverse Transcription Kit. The matrix that was achieved was free of any contamination from geno- 
Table 1. Clinical characteristics of infertile and fertile women

\begin{tabular}{|l|c|c|}
\hline Characteristics & Patients & Controls \\
\hline Number & 15 & 10 \\
\hline Age (years) & $29(22-41)^{\mathrm{a}}$ & $31(26-38)^{\mathrm{a}}$ \\
\hline Parity & NA & $2(1-3)^{\mathrm{a}}$ \\
\hline Duration of infertility (years) & $3(1-5)$ & NA \\
\hline
\end{tabular}

aMedian (range); NA — not applicable

mic DNA, amplification of which could falsify the real time PCR reaction.

\section{Real-time PCR}

\section{Primers design}

Primers for HOXA-10, HOXA-11 and GAPDH were constructed using Primer3 software [18]. Primers for ACTB were designed on the basis of RTPrimerDB [19]. Their specificity for cDNA was verified in the BLAST database [20]. Additionally, the thermodynamic results of designed primers were checked using Oligo Analyzer software to confirm the specificity of achieved primers. Primer sequences, size of achieved reaction products and Tm (melting temperature) results are presented in Table 2.

\section{Real-time PCR reaction}

All reactions were conducted using DyNAmo HS SYBR Green qPCR Kit (Finnzymes, Finland) and Rotor-Gene 3000 thermocycler (Corbett Research, Australia). Specificity of achieved reaction products was assessed on $2 \%$ agarose gel and second derivative of melting curve for the PCR reaction.

To establish the levels of given transcripts in the studied samples, we constructed standard curves generated by the $\mathrm{Ct}$ (value where amplification curve cross- es the threshold line) with seven subsequent ten-fold dilutions of linear DNA, which is the product of a given set of primers. The quality of achieved curves described with $\mathrm{R}^{2}$ was 0.999 and the efficiency $\mathrm{E}$ of the reaction was within the range $1.0-0.97$. The thermal profile was based on the manufacturer's instructions regarding the specific annealing temperature of the primers. Because the use of different reference genes in different tissues is often a subject of debate, we verified the adequacy of ACTB with GAPDH [21]. The expression of both reference genes was highly correlated: the correlation coefficients were 0.89 and 0.96 respectively for the study and control groups $(\mathrm{p}<0.01)$. For each sample, duplicated real-time PCR was processed in the presence of negative and positive controls and no template control.

The calculation of $\mathrm{Ct}$ values for standards, studied samples and construction of standard curves was performed with the Rotor-Gene 3000 software. Additionally, REST2005 software was used to determine the relative expression of HOXA-10 and HOXA-11 in infertile women vs. control subjects. The source of the description of the REST 2005 method can be found in the references [22].

\section{Western blot}

The biopsied part of the endometrium, at least 100 $\mathrm{mg}$, was homogenized in liquid nitrogen and lyzed in RIPA buffer (SIGMA, USA). Next, $30 \mu \mathrm{g}$ of total protein were resuspended in sample buffer and separated on a $12 \%$ tris-glycine gel using SDS-PAGE. Gel proteins were transferred to nitrocellulose (Bio-Rad Laboratories, USA), which was blocked with 5\% milk in tris-buffered saline/tween. Immunodetection was performed with rabbit polyclonal antibody to HOXA-10 (Abcam, USA) and mouse monoclonal antibody

Table 2. Primer sequences, size of achieved reaction products, and Tm (melting temperature) results

\begin{tabular}{|c|c|c|c|}
\hline Gene & Tm $\left[^{\circ} \mathbf{C}\right]$ & Product size $(\mathbf{b p})$ & Sequence \\
\hline $\begin{array}{c}\text { GAPDH } \\
\text { FF }\end{array}$ & 65.3 & 94 & TGCCAAATATGATGACATCAAGAA \\
REV & 67.3 & & GGAGTGGGTGTCGCTGTTG \\
\hline ACTB & 64.2 & 139 & TGAAGGTAGTTTCGTGGATGC \\
FF & 64.1 & & TCCTCCCTGGAGAAGAGCTAC \\
REV & 64.9 & 146 & CTGACTGGGCTGGGTTTG \\
\hline HOXA-10 & 64.9 & & ACCTCAGGCCAGACACCTC \\
FF & & 136 & \\
REV & 64.4 & & CTCAGTGTCTGGCTGCAGAG \\
\hline HOXA-11 & 64.0 & & GCTTCCAAGCTCAGTTCAAGA \\
FF & & & \\
\hline
\end{tabular}


to HOXA-11 (Abcam, USA), followed by incubation with gout anti-rabbit HRP-conjugated antibody ( $\mathrm{Ab}-$ cam, USA) and rabbit anti-mouse HRP-conjugated antibody (Abcam, USA), respectively. The membranes were reblotted and incubated with anti-actin HRP-conjugated antibody (clone I-19) (Santa Cruz, USA) to ensure equal protein loading of the lanes. Bands were revealed using Lumi-Light Western Blotting Substrate (Roche, Germany) and Hyperfilm ECL (Amersham, USA). Kodak Gellogic 200 Imaging System (Carestream, USA) was used to compare optical density of the bands.

\section{Immunohistochemical technique}

Specimens were preserved in buffered formalin $(\mathrm{pH}$ 7.4-7.6), immersed in paraffin blocks and dissected into pieces up to 3-4 $\mu \mathrm{m}$ thick. Eight to ten serial sections were obtained from each sample and mounted on adhesive glass slides. Part of the sections was stained with hematoxylin and eosin $(\mathrm{H}+\mathrm{E})$ and neighboring sections underwent immunohistochemical staining to identify the presence of HOXA-10 and HOXA-11 antigen. Dako LSAB +, System HRP, rabbit polyclonal IgG antibodies were used for the immunoreaction.

\section{Staining procedure}

Epitopes were retrieved from paraffin in a hot water bath with Retrieval Solution buffer at $95-99^{\circ} \mathrm{C}$ for 40 minutes. Endogenous peroxidase was blocked by $3 \%$ $\mathrm{H}_{2} \mathrm{O}_{2}$ for five minutes, then the sections were probed with HOXA-10 or HOXA-11 antibody, rinsed in trisbuffered saline (TBS) twice, incubated with secondary antibody (biotinylated link) for another $30 \mathrm{mi}$ nutes and rinsed again in TBS twice.

The sections were probed with the tertiary antibody (Streptavidin HRP) for 30 minutes and rinsed twice in TBS. Substrate chromagen solution was added for ten minutes and the sections were rinsed in TBS. The sections were stained with $\mathrm{H}+\mathrm{E}$, dehydrated and embedded in Canadian Balm.

Santa Cruz Biotechnology antibodies against HOXA-10 and HOXA-11 diluted 1:200 were used for staining. The staining was visualized and evaluated on an Axioskop 40 microscope, and images were captured using a Canon PowerShot G5.

The degree of HOXA-10, HOXA-11 gene expression was evaluated on the basis of the number of cells with immunopositive reaction observed at 40 $\times$ magnification. Controls for the immunostaining procedure were prepared by omission of the first antibody.
$\mathrm{H}+\mathrm{E}$ staining was used to assess cell cycle phase. According to the criteria of Noyes and Hertig [23], all biopsy specimens were from the middle secretory phase $\left(21^{\text {st }}-23^{\text {rd }}\right.$ day of the cycle). Four randomly chosen parts of tissue from every patient were analyzed. We obtained almost $100 \%$ reaction in the cell nuclei of endometrial stromal cells for the presence of HOXA-10, HOXA-11 protein.

\section{Statistical analysis}

Statistical analysis was conducted using Sigma Stat 3.5.The significance of differences between groups was determined using nonparametric Mann-Whitney Rank Sum test. REST2005 software was used to measure the transcription levels differences. Correlations between HOXA transcripts and proteins levels were assessed by Spearman analysis. $p<0.05$ was considered statistically significant.

\section{Results}

\section{HOXA-10 transcript levels in the endometria of infertile patients and controls}

To compare HOXA-10 transcript levels in the endometria of infertile patients and controls, we used REST2005.

Randomized comparison of Ct showed a statistically significant decreased HOXA-10 transcript level $(p=0.003)$ in infertile patients compared to controls (Table 3).

\section{HOXA-11 transcript levels in the endometria of infertile patients and controls}

Randomized comparison of Ct showed a statistically significant decreased HOXA-11 transcript level ( $\mathrm{p}=$ $=0.012$ ) in the idiopathic infertility group compared to controls (Table 3 ). We did not find a correlation between HOXA-10 and HOXA-11 transcript levels in the study and control groups.

\section{HOXA-10, HOXA-11 protein levels in the endometria of infertile patients and controls}

To compare HOXA-10 and HOXA-11 protein levels, we used Western blotting analysis. There was no significant decrease in HOXA-10 protein level between the infertility and control groups $(p=0.074)$ (Table 4). However, we observed a significantly higher level of HOXA-11 protein in endometria from infertile patients compared to controls ( $\mathrm{p}=$ $=0.015)($ Table 4). 
Table 3. HOXA-10, HOXA-11 transcripts in the endometria of the infertile group compared to the control group

\begin{tabular}{|l|c|c|c|c|c|c|}
\hline Gene & Reaction efficiency & Expression & Std. error & $\mathbf{9 5 \%}$ CI & P(H1) & result \\
\hline HOXA-10 & 0.94 & 0.054 & $0.006-0.411$ & $0.001-1.123$ & 0.003 & Down \\
\hline HOXA-11 & 0.9 & 0.066 & $0.008-0.487$ & $0.000-5.014$ & 0.012 & Down \\
\hline
\end{tabular}

HOXA-10 and HOXA-11 are down-regulated in the infertile group compared to the control group by a mean factor of 0.054 (SE range is $0.006-0.0411), \mathrm{p}=0.003$ and 0.066 (SE range is $0.008-0.487), \mathrm{p}=0.013$, respectively

Table 4. HOXA-10, HOXA-11 protein levels in the endometria of infertile patients and controls. The amount of Western blot-detected proteins was presented as the HOXA-10, HOXA-11 to beta-actin band optical density ratio

\begin{tabular}{|l|c|c|c|c|c|}
\hline & \multicolumn{2}{|c|}{ Infertile group } & \multicolumn{2}{c|}{ Controls } & $\mathbf{p}^{*}$ \\
\hline Gene & Median (range) & Mean $( \pm$ SD) & Median (range) & Mean ( \pm SD) & \\
\hline HOXA-10 & $0.603(0.429-1.23)$ & $0.246 \pm 0.0819$ & $0.809(0.502-1.104)$ & $0.213 \pm 0.0674$ & 0.074 \\
\hline HOXA-11 & $1.460(0.945-4.069)$ & $1.183 \pm 0.394$ & $0.62(0.316-2.127)$ & $0.591 \pm 0.187$ & 0.015 \\
\hline
\end{tabular}

*Mann-Whitney test

We did not find a correlation between HOXA-10 and HOXA-11 protein levels in infertility and control groups. In both groups, we did not find a correlation between HOXA genes transcripts and protein levels.

\section{Immunohistochemical evaluation of HOXA-10 and HOXA-11 protein localization}

Our results showed that HOXA-10 and HOXA-11 proteins were localized in the nuclei of the endometrial stromal cells, whereas the cytoplasm of these cells did not stain (Figure 1). Nuclei of epithelial glandular cells and luminal epithelial cells remained negative. Comparing the immunoreactions in the endometria obtained from infertile women and those obtained from controls, there were some differences (although not statistically significant) in immunoreactions directly under luminal epithelial cells (Figure 1). In six out of 15 women ( $40 \%$ ) with idiopathic infertility, either no immunoreaction in the stromal cells of this part was detected, or only in a very few nuclei. This was true of only one patient ( $10 \%)$ from the control group.

Apart from the described phenomenon, immunohistochemical analyses did not reveal other differences between amounts of HOXA-10 and HOXA-11 protein levels in infertility and control groups. The number of positively stained nuclei of endometrial stromal cells in both groups did not differ (it was 97\%). In ten different fields which were magnified $\times 20,218$ to 650 endometrial stromal cells were found in $1 \mathrm{~mm}^{2}$, containing 212 to 630 positive nuclei. Differences in the numbers of endometrial stromal cells in $1 \mathrm{~mm}^{2}$, and thus the number of positive nuclei, were associated with the luteinization process. In the fields with endometrial edema, the number of immunopositive stromal cells was lower (from 218), compared to fields without edema (to 650). For every 100 counted stromal cells, 2-3 cells were immunonegative, irrespective of the degree of stromal edema. These results indicate that the luteinization process is multifocal and is similar in both groups.

\section{Discussion}

Only very few endometrial receptivity markers are indeed indispensable in the implantation process. Research has revealed that after elimination of several receptivity markers, implantation still occurs [24]. HOXA genes encode transcription factors that are indispensable in the implantation process and, most probably, regulate endometrial development in response to sex steroid hormones [25]. Altered HOXA genes expression has been observed in women with decreased implantation rate. These genes are believed to be receptivity markers and their altered expression may help to identify women with implantation failure [26, 27].

In our study, we compared HOXA-10 and HOXA-11 transcripts and their protein product levels in the endometrium. Using immunohistochemical techniques, we compared the localization of HOXA-10 and HOXA-11 protein in the implantation window in women with idiopathic infertility and in controls.

We found statistically significant lower HOXA10 and HOXA-11 transcript levels in infertile women compared to the control group. Our results were similar to those reported by Matsuzaki et al. [15]. Those authors showed a statistically significantly lower level of HOXA-10 mRNA in endometrial stromal cells in infertile patients compared to normal fertile women. They evaluated groups of patients 
A

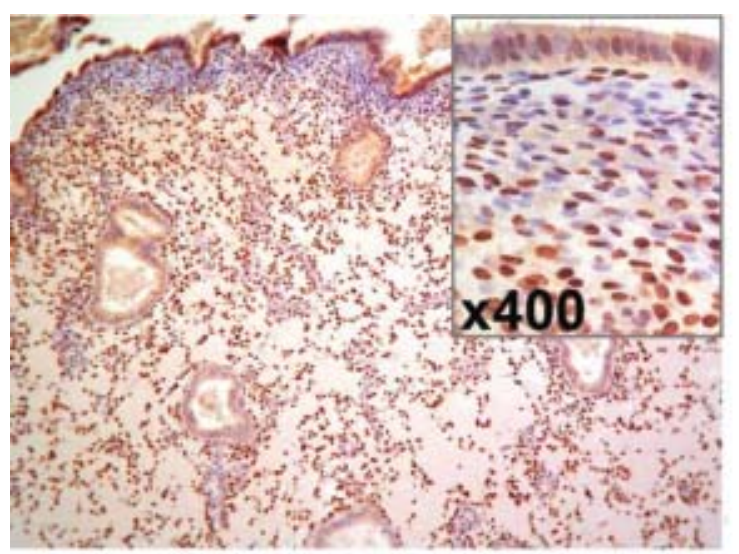

C

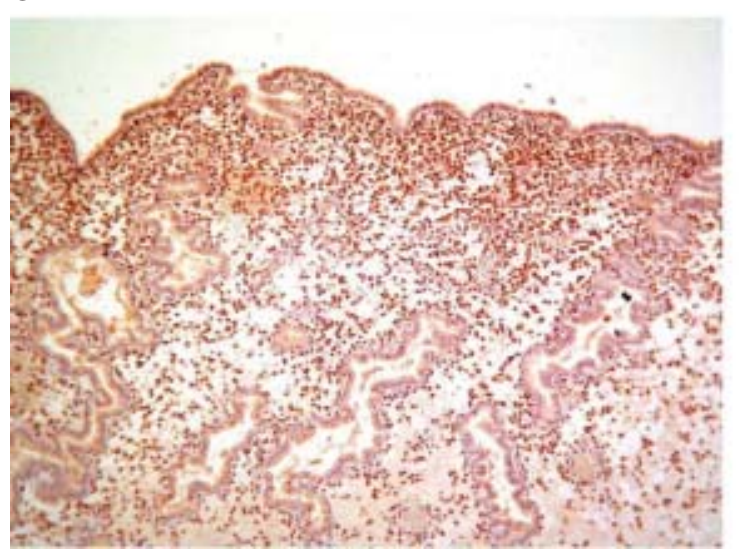

B

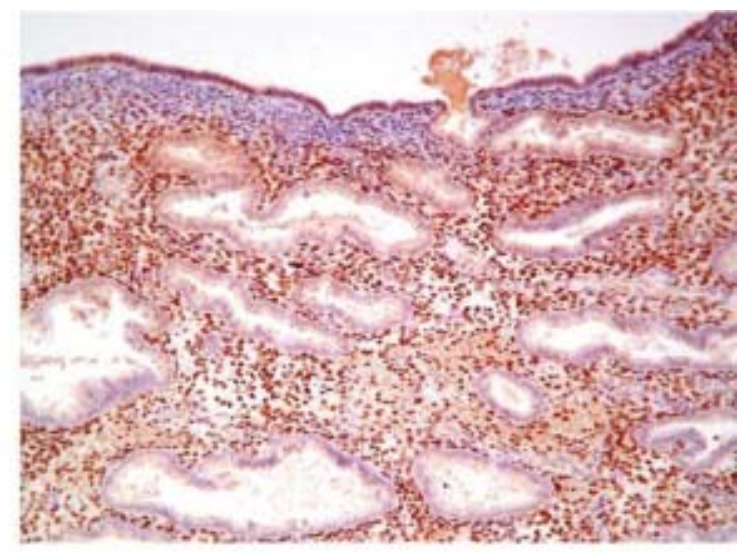

D

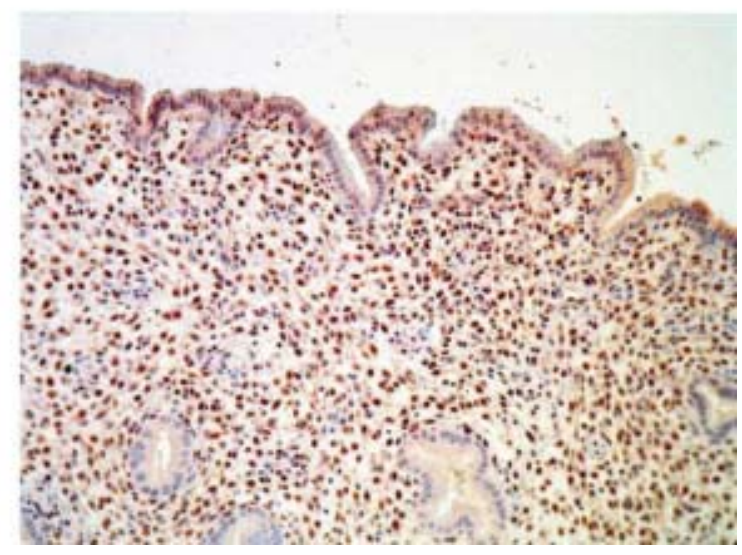

Figure 1. Localization of HOXA-10 and HOXA-11 protein was determined using histochemical staining. The endometrium was obtained on the $21^{\text {st }}-22^{\text {nd }}$ day of the cycle: A. Endometrium from a woman from the idiopathic infertility group, magnification $\times 150$. Immunoreaction to the presence of HOXA-11 protein. Distinct lack of positive immunoreaction in the nuclei of endometrial stromal cells in the area under luminal epithelial cells. Nuclei of endometrial stromal cells and epithelial cells stained blue with hematoxylin are visible in that area. All tissue slides were hematoxylin counterstained. B. Endometrium from a woman from the idiopathic infertility group, magnification $\times 150$. Immunoreaction to the presence of HOXA-10 protein. Localization of immunoreaction similar to HOXA-11. C. Endometrium from a woman from the control group, magnification $\times 150$. Positive immunoreaction to the presence of HOXA- 10 protein in the nuclei of endometrial stromal cells. Reaction is visible in the entire region of the stroma. Negative immunoreaction in epithelial glandular cells and luminal epithelial cells. D. Endometrium from a woman from the control group, magnification $\times 250$. Immunoreaction to the presence of HOXA-11 protein. Localization of immunoreaction similar to HOXA-10

with uterine myomas, endometriosis and idiopathic infertility. Their findings suggest that infertility in these groups of patients may have its source in the altered endometrium function, i.e. lack of its optimal receptivity.

Our study also demonstrated a tendency to lower HOXA-10 protein level in the group of infertile women compared to the control group. Lack of statistical significance may be caused by the small size of the studied groups. By contrast, the level of HOXA-11 protein in the group of infertile women was significantly higher than in normal fertile women. We may formulate a hypothesis that regulation of HOXA-11 expression could process on translation level. This phenomenon takes place in the process of protamin synthesis during spermatogenesis; the transcript level does not correlate with the protein level [28].

Decreased HOXA-10 gene expression level in the endometrium during the implantation window was found in infertile women with PCOS [29] and hydrosalpinx [30]. In the quoted work, gene expression normalized four months after salpingectomy. This suggests that environmental factors could also influence genes expression regulation.

Rackow and Taylor [26], as well as Taylor et al. [27], reported altered HOXA-10 and HOXA-11 expression in infertile women with endometriosis and with uterine myomas. 
We demonstrated, using the immunohistochemical technique, that HOXA-10 and HOXA-11 protein were present in the nuclei of endometrial stromal cells. A similar immunolocalization of HOXA-10 protein was reported by Matsuzaki et al. [15]. According to other authors, cell nuclei and cytoplasm of the stroma, glands, epithelium and blood vessels reveal the presence of HOXA-10 and HOXA-11 protein. These differences in the immunohistochemical protein localization derive from the differing investigation methods applied by various authors.

Lack of immunoreaction in stromal cells located directly under luminal epithelial cells in $40 \%$ of women with idiopathic infertility and $10 \%$ of controls was a particularly interesting observation. This phenomenon may be associated with endometrial inability to get optimal receptivity. Different concentrations of immunopositive cell nuclei in the infertility and control groups indicate that the process of endometrial luteinization is not alike.

Matsuzaki et al. [15] showed statistically significant differences of altered HOXA-10 protein expression between the idiopathic infertility and the control group. In accordance with our observations, the authors concluded that in some patients with idiopathic infertility the HOXA-10 protein expression is not altered. In that group of women, infertility may be caused by a different molecular defect of the endometrium associated with implantation, but these findings need further research.

In both studied groups, no correlation was found between HOXA-10 and HOXA-11 transcript and protein levels. Analysis of the expression of both HOXA genes during the entire menstrual cycle reveals that they increase at the same level, starting with the early luteal phase and with the peak coming during the implantation window [24, 27].

Godbole et al. [8] investigated monkeys and did not confirm the correlation between HOXA-10 and HOXA-11 gene expression in conceptual cycles. High HOXA-10 expression was accompanied by low (comparable to the control group) HOXA-11 expression. This might support the theory that both genes are regulated by different mechanisms during implantation.

The mechanism of decreased HOXA-10 and HOXA-11 gene expression in the endometrium remains a popular topic of discussion. The methylation of promotor sequences could be responsible for decreased transcript levels. Wu et al. [31] have shown altered methylation of the HOXA-10 gene in women with advanced endometriosis. On the basis of research carried out on animal models by Kim et al. [2] and Lee et al. [32], it is possible to conclude that en- dometriosis induces DNA methylation and altered gene expression in the eutopic endometrium. Computational analysis of promoter sequence of HOXA genes shows the presence of many transcription factor-binding sites e.g. Sp1, NF-1, E2F, LSF [33]. Methylation of these sites inhibits the binding of transcription factors to their binding sites within promotor sequence.

Our results suggest that HOXA-10 and HOXA11 gene expression in the endometrium during the implantation window may not be altered in patients with idiopathic infertility. Further research is needed to investigate what molecular mechanism impairs the optimal implantation conditions in this group of women.

\section{Acknowledgements}

This study was supported by Ministry of Science and Higher Education grant NN407 162633, and UE grant 162/E-392/CD/DFS-4/2004.

\section{References}

1. Adamson GD, Baker VL. Subfertility: causes, treatment and outcome. Best Pract Res Clin Obstet Gynaecol. 2003;17:169-185 .

2. Kim JJ, Taylor HS, Ladhani O, et al. Altered expression of HOXA10 in endometriosis: potential role in decidualization. Mol Hum Reprod. 2007;13:323-332.

3. Mikolajczyk M, Wirstlein P, Skrzypczak J. The impact of leukemia inhibitory factor in uterine flushing on reproductive potential of infertile women - a prospective study. Am J Reprod Immonol. 2007;58:65-74.

4. Salih S, Taylor H. HOXA10 gene expression in human fallopian tube and ectopic pregnancy. Am J Obstet Gynecol. 2004;190:1404-1406.

5. Skrzypczak J, Wirstlein P, Mikołajczyk M, Ludwikowski G, Zak T. TGF superfamily and MMP2, MMP9, TIMP1 genes expression in the endometrium of women with impaired reproduction. Folia Histochem Cytobiol. 2007;45: $143-148$.

6. Skrzypczak J, Wirstlein P, Mikołajczyk M. Could the defects in the endometrial extracellular matrix during the implantation be a cause of impaired fertility? Am J Reprod Immunol. 2007;57:40-48.

7. Skrzypczak J, Wirstlein P, Mikołajczyk M. Is glycodelin an important marker of endometrial receptivity? Gin Pol. 2005;76:770-781.

8. Godbole GB, Modi DN, Puri CP. Regulation of homebox A 10 expression in the primate endometrium by progesterone and embryonic stimuli. Reproduction. 2007;134:513-523.

9. Daftary GS and Taylor HS. Endocrine regulation of HOX genes. Endocrine Reviews. 2006;27:331-355.

10. Bagot CN, Troy HS, Taylor HS. Alteration of maternal HOXA10m RNA expression by in vivo gene transfection affects implantation. Gene Ther. 2000;7:1378-1384.

11. Benson GV, Lim H, Paria BC. Mechanisms of reduced fertility in Hoxa-10 mutant mice: uterine homeostasis and loss of maternal Hoxa-10 expression. Development. 1996;58: 337-347. 
12. Li S, Ma L, Chiang T, Burow M et al. Promoter CpG methylation of Hox-a10 and Hox-a11 in mouse uterus not altered upon neonatal diethylstilbestrol exposure. Mol Carcinog. 2001;32:213-219.

13. Lalwani $\mathrm{S}, \mathrm{Wu} \mathrm{H}$, Reindollar RH, Gray MR. HOXA $10 \mathrm{mu}-$ tations in congenital absence of uterus and vagina. Fertil Steril. 2008;89:325-330.

14. Lu Z, Hardt J, Kim JJ. Global analysis of genes regulated by HOXA10 in decidualization reveals a role in cell proliferation. Mol Hum Reprod. 2008;14:357-366.

15. Matsuzaki S, Canis M, Darcha C, Pouly J, Mage G. HOXA-10 expression in midsecretory endometrium of infertile patients with either endometriosis, uterine fibromas or unexplained infertility. Hum Rep. 2009;24:3180$-3187$.

16. Gui Y, Zhang J, Yuan L, Lessey BA. Regulation of HOXA-10 and its expression in normal and abnormal endometrium. Mol Hum Reprod. 1999;5:866-873.

17. Kao LC, Tulac S, Lobo S, Imani B, Yang JP, Germeyer A, Osteen K, Taylor RN, Lessey AA, Giudice LC. Global gene profiling in human endometrium during the window of implantation. Endocrinology. 2002;143:2119-2138.

18. http://frodo.wi.mit.edu/primer3/. Accessed: February 10, 2010.

19. http://medgen.ugent.be/rtprimerdb/assay_report.php? RTPrimerDB_ID=771. Accessed: February 10, 2010.

20. http://blast.ncbi.nlm.nih.gov/Blast.cgi. Accessed: February 10, 2010.

21. Romanowski T, Markiewicz A, Bednarz N, Bielawski KP. Housekeeping genes as a reference in quantitative real-time RT-PCR. Postepy Hig Med Dosw. 2007;61:500-510.

22. http://www.gene-quantification.de/REST-2005_manual_ 119.12.pdf. Accessed: February 10, 2010.
23. Noyes RW, Hertig AT, Rock J. Dating the endometrial biopsy. Fertil Steril. 1950;1:3-25.

24. Kwon H., Taylor HS. The role of HOX genes in human implantation. NY Acad Sci. 2004;1034:1-18.

25. Taylor HS, Arici A, Olive D, Igarashi P. HOXA10 is expressed in response to sex steroids at the time of implantation in the human endometrium. J Clin Invest. 1998;10:1379-1384.

26. Rackow BW, Taylor HS. Submucosal uterine leiomyomas have a global effect on molecular determinants of endometrial receptivity. Fertil Steril. 12 Jun 2008; PMID: 18555231.

27. Taylor HS, Bagot C, Kardana A, Olive D, Arici A. HOX gene expression is altered in endometrium of women with endometriosis. Hum Reprod. 1999;14:1328-1331.

28. Carrell DT, Emery BR, Hammoud S. Altered protamine expression and diminished spermatogenesis: what is the link? Hum Reprod Update. 2007;13:313-27. Epub 2007 Jan 5.

29. Cermik D, Selam B, Taylor HS. Regulation of HOXA-10 expression by testosterone in vitro and in endometrium of patients with polycystic ovary syndrome.J Clin Endocrinol Metab. 2003;88:238-243.

30. Daftary GS, Kayisli U, Seli E, Bukulmez O, Arici A, Taylor HS. Salpingectomy increases peri-implantation endometrial HOXA-10 expression in women with hydrosalpinx. Fertil Steril. 2007;87:367-372.

31. Wu Y, Halverson G, Basir Z, Strawn E, Yan P, Guo SW. Aberrant methylation at HOXA 10 may be responsible for its aberrant expression in the endometrium of patients with endometriosis. Am J Obstet Gynecol. 2005;193:371-380.

32. Lee B, Du H, Taylor HS. Experimental murine endometriosis induces DNA methylation and altered gene expression in eutopic endometrium. Biol Reprod. 2009;80:79-85.

33. http://zlab.bu.edu/ $\sim$ mfrith/cister.shtml. Accessed: February $10,2010$. 